\title{
Anatomy education: suddenly back to the future?
}

\author{
F. Duparc ${ }^{1}\left[\right.$. B. Grignon ${ }^{2}$
}

Published online: 5 January 2022

(C) The Author(s), under exclusive licence to Springer-Verlag France SAS, part of Springer Nature 2022

2021 is over, but it has been a second very difficult year, in the fields of anatomy teaching and anatomical research.

In the first part of this issue, some already well-known modern educational tools have been reevaluated, while more profound changes due to the pandemic have been investigated.

We aimed to open the debate with an article about the quality of the teaching models, high or low-fidelity, but with a common goal: the anatomical exactness.

In the undergraduate period, despite the using of 3D-models looks very useful, the difficulty is also related to who can teach. The role of radiology in anatomy teaching, as well as the role of radiologists in anatomy teaching has also been revisited.

Another concept, the student-centered education is also developed in this issue, leading to a focusing on the individual learning, with questioning about the teacher-centered usual concept.

However, despite the growing place of the 3D virtual dissection for undergraduate students, the influence of dissection for the acquisition of surgical skills has been reevaluated and strongly supported, at the step before training of simulators, for trainees in surgery.

The Covid-19 pandemic has jostled the anatomical education with a sudden, rapid and quite violent manner.

The classical face-to-face education has been interrupted, leading the anatomy teachers and the students to be transformed to face-to-screen and the possibilities of adaptation have been studied, on each side of this new interface. One

\section{F. Duparc}

fabrice.duparc@univ-rouen.fr

B. Grignon

b.grignon@chu-nancy.fr

1 Department of Anatomy, Faculty of Medicine-Pharmacy, University of Rouen-Normandy, 22 Boulevard Gambetta, 76183 Rouen, France

2 Department of Anatomy, Faculty of Medicine, University of Lorraine, Avenue de la Forêt de Haye, 54505 Vandœuvre-Lès-Nancy, France could have consider it was a generational evolution, but it achieved within few weeks but not through a decade or more. The e-learning, through a large span of modes, web-based, virtual classroom, virtual dissection, but also virtual evaluation, could save the major part of the anatomical education. New modalities should offer both educational material and self-teaching tools, and Dissection Educational Videos provided an interesting way for distance teaching.

The evaluation of distance anatomy education has been studied in four articles, regarding the students' perception and perspectives. A review of the changes submits a state of the art of the different tools that were used.

The pandemic definitively changed anatomy education, and we need to anticipate the post-pandemic improvements we shall be able to install, with taking into account the incredible lessons we had to face and conciliating the e-Anatomy, the return to face-to-face teaching, and the necessary dissection of the human body.

It is sure that the effects of the pandemics about our ways to teach anatomy and perform researches will also continue to strongly influence the research in the field of clinical anatomy.

At least, a study states the question about the so-called "inadequate knowledge" in Anatomy and the "hazardous" effect on "successful" clinical practice: the conclusions support the necessary re-enforcement of our fields of activity in clinical anatomy.

The second part of this issue compiled some articles about the thorax anatomy, and rare but clinically important anatomical variations of the hepatic arteries.

Our warmest thanks are addressed to the Associate Editors and the Reviewers who made a huge job for managing about 900 submissions in 2021.

We wish you a very Happy New Year 2022.

F. Duparc and B. Grignon, Editors in chief. 
Publisher's Note Springer Nature remains neutral with regard to jurisdictional claims in published maps and institutional affiliations. 